Pacific Journal of Mathematics

SYSTEMS OF FUNCTIONAL DIFFERENTIAL INEQUALITIES 


\title{
SYSTEMS OF FUNCTIONAL DIFFERENTIAL INEQUALITIES AND FUNCTIONAL DIFFERENTIAL SYSTEMS
}

\author{
G. S. LADDE
}

In this paper, by employing the theory of systems of functional differential inequalities, a very general comparison theorem for functional differential systems in the context of vector Lyapunov functions is developed. Furthermore, this comparison theorem has been applied to derive sufficient conditions for stability of the equilibrium state of the functional differential systems under structural perturbations caused by the interactions among the states of the system. Finally, the role of comparison theorem in the framework of vector Lyapunov functions has been demonstrated by investigating the stability analysis of hereditary interconnected systems.

One of the most versatile techniques in the theory of nonlinear differential equations is the second method of Lyapunov. This method has been successfully employed to study a variety of problems, in a unified way, of ordinary differential equations, functional differential equations (deterministic and stochastic), partial differential equations and differential equations in abstract spaces. A systematic and unified development of the theory and applications of differential inequalities in the context of Lyapunov's second method can be found in a recent monograph of Lakshmikantham and Leela [6].

Most of the results that have appeared for functional differential systems, are in the framework of single Lyapunov functions. Therefore, it is natural to expect its extension in the context of vector Lyapunov functions. In certain situations, the use of vector Lyapunov functions is more advantageous over the single Lyapunov functions (Lakshmikantham and Leela [6], and Ladde [4]).

The obtained comparison theorems in the context of vector Lyapunov functions and the theory of systems of functional differential inequalities have been utilized to study the stability analysis of hereditary interconnected systems. As a by-product of this analysis the presented results extend the earlier results with regard to ordinary interconnected systems, for example, results due to Bailey [1], Grujic and Šiljak [2], and Siljak [8,9]. Furthermore, these results provide an alternative approach to the use of either vector Lyapunov functionals, or vector Lyapunov functions in the context of a minimal class of functions [11]. 
2. Notation and definition. Let $R^{n}$ denote the $n$ dimensional Euclidean space with any convenient norm $\|-\|$. Let $\boldsymbol{R}_{+}$ denote the nonnegative real line while $R$ will be used, as usual for $R^{1}$. Given $\tau>0$, let $\mathscr{C}^{n}=C\left[[-\tau, 0], R^{n}\right]$ denote the space of continuous functions with domain $[-\tau, 0]$ and range in $R^{n}$. For $\phi \in \mathscr{C}^{n}$, we define $\|\phi\|_{0}=\sup _{-\tau \leqq s \leqq 0}\|\phi(s)\|$. Let $(\cdot)^{T}$ denote the transpose of a vector or a matrix. Suppose that $x \in C\left[[-\tau, \infty), R^{n}\right]$. For $t \in R_{+}$, let $x_{t}$ denote a translation of the restriction of $x$ to the interval $[t-\tau, t]$; more specifically, $x_{t}$ is an element of $\mathscr{C}^{n}$ defined by $x_{t}(s)=x(t+s)$, $-\tau \leqq s \leqq 0$. Let $\mathscr{C}_{+}^{n}$ denote the set of all $\phi \in \mathscr{C}^{n}$ such that $\phi(s) \geqq 0$ on $[-\tau, 0]$. For $x \in R^{n}$, let $x=\left(\left(x^{1}\right)^{T},\left(x^{2}\right)^{T}, \cdots,\left(x^{m}\right)^{T}\right)^{T}$, and for $\phi \in \mathscr{C}^{n}$, let $\phi_{m}=\left((\phi)^{T}, \cdots,\left(\phi^{m}\right)^{T}\right)^{T}$, where $x^{i} \in R^{n_{i}}, \phi^{i} \in \mathscr{C}^{n_{i}},\left(\phi^{i}\right)^{T}=\left(\phi^{i}(s)\right)^{T}$ on $[-\tau, 0]$, and $\sum_{l=1}^{m} n_{l}=n$. For $\rho_{i}>0$, let $C_{\rho}=\left\{\phi \in \mathscr{C}^{n}:\left\|\phi^{i}\right\|_{0}<\rho_{l}, i \in I\right\}$ and $S_{\rho}=\left\{x \in R^{n}:\left\|x^{i}\right\|<\rho_{\imath}, i \in I\right\}$, where $I=\{1,2,3, \cdots, m\}$.

Consider the system of functional differential equations

$$
\dot{x}=f\left(t, x, x_{t}\right), \quad x_{t o}=\phi_{0}
$$

where $x \in R^{n}, f \in C\left[R_{+} x S_{\rho} x C \rho, R^{n}\right]$, and $f$ is smooth enough to guarantee the existence of solutions of (1) for $t \geqq t_{0}$. For existence theorems, see Lakshmikantham and Leela [6]. We shall assume that $f(t, 0,0) \equiv 0$, so that the system (1) possesses a trivial solution. Let $x\left(t_{0}, \phi_{0}\right)(t)$ be any solution of (1) with an initial function $\phi_{0} \in C_{\rho}$ at $t=t_{0}$.

Now, we decompose (1) into $m$ interconnected subsystems described by the equations

$$
\dot{x}^{\imath}=h_{\imath}\left(t, x^{\imath}, x_{t}^{t}\right)+H_{\imath}\left(t, x, x_{t}\right), \quad i \in I
$$

where $x_{i} \in R^{n}$ is the state of the $i$ th subsystem, and represents the $i$ th component of the state vector $x$ and $h_{t} \in C\left[R_{+} \times S_{\rho_{i}} \times C \rho_{l}, R^{n}\right]$, and $H_{t} \in C\left[R_{+} \times S_{\rho} \times C_{\rho}, R^{n_{i}}\right]$, where $S_{\rho_{t}}=\left\{x^{i} \in R^{n_{i}}:\left\|x^{\imath}\right\|<\rho_{l}\right\}$, and $C_{\rho_{i}}=$ $\left\{\phi^{i} \in \mathscr{C}^{n} i:\left\|\phi^{\prime}\right\|_{0}<\rho_{i}\right\}$.

We assume that interconnection functions $H_{t}\left(t, x, x_{t}\right)$ depend on the $m \times m$ interconnection matrices $E=\left(\boldsymbol{e}^{i j}\right)$ and $L_{t}=\left(\ell_{t}^{i t}\right)$

$$
H_{t}\left(t, x, x_{t}\right)=H_{t}\left(t, e^{t_{1}} x^{1}, e^{t_{2}} x^{2}, \cdots, e^{t m} x^{m}, \ell_{t}^{i_{1}} x_{t}^{1}, \ell_{t}^{i_{2}} x_{t}^{2}, \cdots, \ell_{t}^{i m} x_{t}^{m}\right)
$$

for $i \in I$, where $\boldsymbol{e}^{i j} \in C\left[R_{+},[0,1]\right]$ are coupling functions which are elements of $m \times m$ ordinary interconnection matrix function $E(t)$, and for $t \in R_{+}, \quad \ell_{t}^{\prime \prime} \in C[[-\tau, 0],[0,1]]$ are coupling functions which are elements of $m \times m$ hereditary interconnection matrix function $L_{t} ; \ell_{t}^{i j} x_{t}^{i}=$ $\ell^{\prime \prime}(t+s) x^{\prime}(t+s)$ for $s \in[-\tau, 0]$.

When $E(t) \equiv 0 \equiv L_{t}(s)$, from (2), we get the free or isolated subsystems described by the differential equations.

$$
\dot{x}^{\imath}=h_{t}\left(t, x^{i}, x_{t}^{\imath}\right), \quad i \in I
$$


where $h_{i}(t, 0,0) \equiv 0$, and $x^{i}=0$ is the unique equilibrium of the $i$ th free subsystem.

Definition 1. The trivial solution $x=0$ of the system (1) is connectively asymptotically stable if and only if it is asymptotically stable in the sense of Lyapunov for all interconnection matrices $E(t), L_{t}$, i.e., for each $\epsilon>0, t \in R_{+}$, there exists a $\delta>0$ such that $\left\|\phi_{0}\right\|_{0}<\delta$ implies $\left\|x\left(t_{0}, \phi_{0}\right)(t)\right\|<\epsilon$ for all $t \geqq t_{0}$, and there is a $\delta_{0}>0$ such that $\left\|\phi_{0}\right\| \leqq \delta_{0}$ implies $\left\|x\left(t_{0}, \phi_{0}\right)(t)\right\| \rightarrow 0$ as $t \rightarrow \infty$, for all interconnection matrices $E(t)$, $L_{t}$.

Definition 2. The equilibrium $x=0$ of the system (1) is exponentially connectively stable if and only if there exist two positive numbers $a$ and $K=K(\rho)$ such that

$$
\left\|x\left(t_{0}, \phi_{0}\right)(t)\right\| \leqq K\left\|\phi_{0}\right\|_{0} \exp \left[-a\left(t-t_{0}\right)\right], \quad t \geqq t_{0} .
$$

Note that other stability and boundedness notions (Lakshmikantham and Leela [6]) can be formulated analogously.

Definition 3. A function $a(r)$ is said to belong to the class $K$ if $a \in C\left[R_{+}, R_{+}\right], a(0)=0, a(r)$ is strictly increasing in $r$.

For deriving the connective stability conditions, we need the definition of fundamental interconnection matrices $\bar{E}$ and $\bar{L}_{t}$ in which the constant elements $\overline{\boldsymbol{e}}^{i j}$ and $\overline{\boldsymbol{e}}_{t}^{i j}$ take on binary values 0 or 1 . In case of $\bar{E}$, $\overline{\boldsymbol{e}}^{\prime \prime}$ is 1 if the $j$ th subsystem state $x^{\prime}$ interacts the $i$ th subsystem state $x^{\prime}$, and it is 0 if $x^{j}$ does not interact $x^{\prime}$. Similarly, $\overline{\boldsymbol{e}}_{t}^{i j}$ is 1 if the past state of the $j$ th subsystem $x^{j}$ influences the $i$ th subsystem state $x^{i}$, and it is 0 if $x^{\prime}$ has no influence on the $i$ th subsystem $x^{\prime}$. Further note that the stability conditions are derived for the fundamental interconnection matrices $\bar{E}$, $\overline{L_{t}}$, but are valid for all interconnection matrices $\bar{E}(t), \bar{L}_{t}$, as required in Definitions 1 and 2. Note that the symbols 0,1 are used to denote real numbers as well as functions defined on $[-\tau, 0]$.

REMARK 1. Note that the notion of connective stability includes the usual Lyapunov stability, whenever $E(t)=\bar{E}$ and $\bar{L}_{t}=\bar{L}_{t}$. In other words, the interactions within the subsystems do not vary during the operation of the system, i.e., the interactions depend on initial fixed connections among the subsystems.

3. Comparison principle. In this section, by employing the concept of vector Lyapunov function, and the theory of functional differential inequalities, we develop comparison theorems for the system 
(1). Our results can be considered as extension of the earlier scalar results, Lakshmikantham and Leela [6]. tional

Let $V \in C\left[[-\tau, \infty) \times S \rho, R_{+}^{m}\right]$, and $\phi \in C_{\rho}$. We define vector func-

$$
D_{(1)}^{+} V(t, \phi(0), \phi)
$$

$$
=\lim _{h \rightarrow 0^{+}} \sup \frac{1}{h}[V(t+h, \phi(0)+h f(t, \phi(0), \phi))-V(t, \phi(0)] .
$$

On the basis of the comparison theorem for systems of functional differential inequalities developed by Shendge [10], we now prove the following result which plays an important role in studying the behavior of solutions of functional differential systems.

THEOREM 1. Assume that there exist functions $V(t, x)$ and $g(t, \sigma(0), \sigma)$ satisfying the following conditions:

(i) $\quad V \in C\left[[-\tau, \infty) \times S \rho, R_{+}^{m}\right]$;

(ii) $V(t, x)$ is locally Lipschitzian in $x$, for each $t \in R_{+}$;

(iii) $g \in C\left[R_{+} \times R_{+} \times \mathscr{C}_{+}^{m}, R_{+}^{m}\right]$,

$g(t, \sigma(0), \sigma)$ is quasimonotone nondecreasing in $\sigma(0)$ and nondecreasing in $\sigma$ for each $t \in R_{+}$, and

(6)

$$
D_{(1)}^{+} V(t, \phi(0), \phi) \leqq g\left(t, V(t, \phi(0)), V_{t}\right), \quad \text { for all } \quad(t, \phi) \in R_{+} \times C \rho
$$

and for all interconnection matrices $E(t), L_{t}$, where $V_{t}=V(t+s, \phi(s))$, $-\tau \leqq s \leqq 0$;

(iv) let $r\left(t_{0}, \sigma_{0}\right)(t)$ be the maximal solution of the functional differential system

$$
\dot{u}=g\left(t, u, u_{t}\right), \quad u_{t_{0}}=\sigma_{0},
$$

existing for $t \geqq t_{0}, t_{0} \in R_{+}$.

If $x\left(t_{0}, \phi_{0}\right)(t)$ is any solution of (1) existing for $t \geqq t_{0}$ such that

$$
V_{\mathfrak{k}} \leqq \sigma_{0}
$$

then

$$
V\left(t, x\left(t_{0}, \phi_{0}\right)(t)\right) \leqq r\left(t_{0}, \sigma_{0}\right)(t), \quad t \geqq t_{0},
$$

and all interconnection matrices $E(t), L_{t}$. 
Proof. Let $x\left(t_{0}, \phi_{0}\right)(t)$ be any solution of (1) satisfying (8), and $x_{t}\left(t_{0}, \phi_{0}\right) \in C_{\rho}$. Set $\phi=x_{t}\left(t_{0}, \phi_{0}\right)$, which implies that $\phi(0)=x\left(t_{0}, \phi_{0}\right)$ $(t)$. Define

$$
m(t)=V\left(t, x\left(t_{0}, \phi_{0}\right)(t)\right)
$$

so that

$$
m_{t}=V(t+s, \phi(s))
$$

Since (8) holds, we have $m_{\iota} \leqq \sigma_{0}$. For sufficiently small $h \geqq 0$, we have

$$
\begin{aligned}
m(t+h)-m(t)= & V\left(t+h, x\left(t_{0}, \phi_{0}\right)(t+h)\right)-V\left(t, x\left(t_{0}, \phi_{0}\right)(t)\right) \\
= & V(t+h, \phi(0)+h f(t, \phi(0), \phi))-V(t, \phi(0)) \\
& +V\left(t+h, x\left(t_{0}, \phi_{0}\right)(t+h)\right) \\
& -V(t+h, \phi(0)+h f(t, \phi(0), \phi)) .
\end{aligned}
$$

This together with the hypotheses (i), (ii) and (iii) yields the inequality

$$
D^{+} m(t) \leqq g\left(t, m(t), m_{t}\right), \quad \text { for } \quad t \geqq t_{0} .
$$

From an application of Corollary 4 in Shendge [10] in the context of Remark 3 in [10], we deduce that

$$
V\left(t, x\left(t_{0}, \phi_{0}\right)(t)\right) \leqq r\left(t_{0}, \sigma_{0}\right)(t), \quad t \geqq t_{0} .
$$

Since the inequality (6) is valid for all $E(t)$ and $L_{t}$, so also (11). This completes the proof of the theorem.

The following variant of Theorem 1 is often more useful in applications.

THEOREM 2. Let the hypotheses of Theorem 1 hold except that the inequality (6) is replaced by

$$
\begin{gathered}
A(t) D_{(1)}^{+} V(t, \phi(0), \phi)+\dot{A}(t) V(t, \phi(0)) \\
\leqq g\left(t, A(t) V(t, \phi(0)),(A V)_{t}\right),
\end{gathered}
$$

for $(t, \phi) \in R_{+} \times C \rho$, and for all $E(t), L_{t}$, where $A(t)$ is an $m \times m$ continuously differentiable matrix function defined on $[\tau, \infty)$ with values in $R^{m^{2}}$ such that $\|A(t)\| \rightarrow \infty$ as $t \rightarrow \infty$ and the matrix $A^{-1}(t) \dot{A}(t)$ has nonnegative off-diagonal elements for $t \geqq 0 ; \quad(A V)_{t}=$ $A(t+s) V(t+s, \phi(s))$ for $-\tau \leqq s \leqq 0$. Then, 


$$
(A V)_{\mathfrak{t}} \leqq \sigma_{0}
$$

implies

$$
V\left(t, x\left(t_{0}, \phi_{0}\right)(t)\right) \leqq R\left(t_{0}, \psi_{0}\right)(t), \quad t \geqq t_{0}
$$

where $R\left(t_{0}, \psi_{0}\right)(t)$ is the maximal solution of the differential system

$$
\dot{v}=-A^{-1}(t) \dot{A}(t) v+A^{-1}(t) g\left(t, A(t) v,(A V)_{t}\right), \quad v_{t 0}=\psi_{0},
$$

existing for $t \geqq t_{0}$.

Proof. Setting

$$
W(t, \phi(0))=A(t) V(t, \phi(0)) .
$$

From (12), it is easy to see that

$$
\begin{aligned}
D_{(1)}^{+} W(t, \phi(0), \phi) & =A(t) D_{(1)}^{+} V(t, \phi(0), \phi)+\dot{A}(t) V(t, \phi(0)) \\
& \leqq g\left(t, W(t, \phi(0)), W_{t}\right) .
\end{aligned}
$$

This shows that $W(t, \phi(0))$ satisfies all the hypotheses of Theorem 1 , and consequently, we have

$$
W\left(t, x\left(t_{0}, \phi_{0}\right)(t)\right) \leqq r\left(t_{0}, \sigma_{0}\right)(t), \quad t \geqq t_{0},
$$

where $r\left(t_{0}, \sigma_{0}\right)(t)$ is the maximal solution of (7). It is easy to verify that $r\left(t_{0}, \sigma_{0}\right)(t)=A(t) R\left(t_{0}, \psi_{0}\right)(t)$ with $A\left(t_{0}+s\right) \psi_{0}(s)=\sigma_{0}(s)$. This together with (16) and the definition $W$ yields the desired inequality (14).

REMARK 2. Theorem 1 is generalization of Theorem 8.1.4 in [6], and it is an extension of the result 4.1.1 in [6].

4. Connective stability conditions. In this section, by employing the comparison theorems developed in the preceding section, we shall present a result giving sufficient conditions for connective stability. Other results can be formulated analogously.

Theorem 3. Let the hypotheses of Theorem 1 hold. Further assume that

$$
a(\|x\|) \leqq \sum_{i=1}^{m} V_{i}(t, x) \leqq b(\|x\|), \quad(t, x) \in R \times S_{\rho}
$$

$$
g(t, 0,0) \equiv 0, \quad t \in R_{+} .
$$


Then the stability or asymptotic stability of the trivial solution $u \equiv 0$ of (7) implies the connective stability or connective asymptotic stability of the trivial solution of the system (1).

Proof. Let $x\left(t_{0}, \Phi_{0}\right)(t)$ be any solution of (1) existing for $t \geqq t_{0}$ such that $V\left(t_{0}+s, \Phi_{0}(s)\right) \leqq \sigma_{0}(s)$. Hence, by Theorem 1, we have the following inequality

$$
V\left(t, x\left(t_{0}, x_{0}\right)(t)\right) \leqq r\left(t_{0}, \sigma_{0}\right)(t), \quad t \geqq t_{0},
$$

which implies

$$
\sum_{i=1}^{m} V_{l}\left(t, x\left(t_{0}, \phi_{0}\right)(t)\right) \leqq \sum_{i=1}^{m} r_{i}\left(t_{0}, \sigma_{0}\right)(t), \quad t \geqq t_{0} .
$$

For $i \in I$, let $\rho_{\imath}>\epsilon>0, t_{0} \in R_{+}$be given. Assume that the trivial solution of (7) is stable. Then $a(\epsilon) / \sqrt{m}>0, t_{0} \in R_{+}$, there exists a $\delta_{1}>0$ such that $\left\|\sigma_{0}\right\|_{0} \leqq \delta_{1}$ implies

$$
\left\|r\left(t_{0}, \phi_{0}\right)(t)\right\|<a(\epsilon), \quad t \geqq t_{0} .
$$

Choose $\delta>0$ such that $\delta=b^{-1}\left(\delta_{1}\right)$ and let $\left\|\phi_{0}\right\| \leqq \delta$.

From (18) and (19), we have

$a\left(\left\|x\left(t_{0}, \phi_{0}\right)(t)\right\|\right) \leqq \sum_{i=1}^{m} V_{i}\left(t, x\left(t_{0}, \phi_{0}\right)(t)\right) \leqq \sum_{i=1}^{m} r_{i}\left(t_{0}, \sigma_{0}\right)(t)<a(\epsilon), \quad t \geqq t_{0}$

which implies that

$$
\left\|x\left(t_{0}, \phi_{0}\right)(t)\right\|<\epsilon, \quad t \geqq t_{0}
$$

whenever

$$
\left\|\phi_{0}\right\|_{0} \leqq \delta
$$

Since (20) is valid for all interconnection matrices $E(t), L_{t}$, the stability is indeed connective. The proof of the connective asymptotic stability of the trivial solution of (1) can be formulated analogously.

Remark 3. Theorem 3 can be extended in order to study conditional stability and conditional boundedness concepts (Lakshmikantham and Leela [6]) for functional differential systems. The importance of this theorem lies in the fact that we can obtain stability results for a higher order systems (order $n$ ) by knowing the stability results of lower order systems (order $m$ ). Furthermore, the dimension of a lower order is the same as the dimension of the vector Lyapunov functions. 
5. Applications. The results obtained in the previous sections are applied to study the complex system (1) that is formed by the interconnection of simple isolated subsystems (4). The main idea is the following: by decomposing a complex system into simpler and suitable units, the stability of each unit is aggregated by a single Lyapunov function, and an auxiliary or comparison system is formed on the basis of the nature of interactions among the units of a system. Then, the stability of the overall system is obtained by testing the stability of the auxiliary system.

For each subsystem (4), we assume that there exists a scalar Lyapunov function $V_{l}:[-\tau, \infty) \times S_{\rho_{l}} \rightarrow R_{+}$. Further we assume that $V_{l}$, and interconnection functions $H_{t}$ in (2) satisfy the following conditions: ties:

$\mathrm{C}_{1}$. For each $i \in I$, the function $V_{\imath}$ satisfies the following proper-

(i) $\quad V_{\imath} C\left[[-\tau, \infty) \times S_{\rho}, R_{+}\right]$;

(ii) for $\left(t, x^{t}\right),\left(t, y^{\prime}\right) \in R_{+} \times S_{\rho_{\text {t }}}$

$$
\left|V_{\iota}\left(t, x^{\imath}\right)-V_{\iota}\left(t, y^{\imath}\right)\right| \leqq K_{\iota}\left\|x^{i}-y^{\imath}\right\|
$$

where $K_{t}=K\left(\rho_{\imath}\right)>0$ is a constant

(iii) for $\left(t, x^{\prime}\right) \in R_{+} \times S_{\rho_{t}}$

$$
a_{l}\left(\left\|x^{i}\right\|\right) \leqq V_{l}\left(t, x^{\prime}\right) \leqq b_{\imath}\left(\left\|x^{\imath}\right\|\right)
$$

where $a_{t}, b_{\imath} \in K$;

(iv) for $\left(t, \phi^{i}\right) \in R_{+} \times C_{\rho_{1}}$

$$
\begin{aligned}
D_{(4)}^{+} V_{\imath}\left(t, \phi^{\prime}(0), \phi^{\imath}\right) \leqq & -\alpha_{\imath}\left(V_{\imath}\left(t, \phi^{\prime}(0)\right)\right) w_{\imath}^{1}\left(V_{\imath}\left(t, \phi^{\imath}(0)\right)\right) \\
& +\beta^{\imath}\left(V_{t}^{\imath}\right) w_{\imath}^{2}\left(V_{t}^{\imath}\right),
\end{aligned}
$$

where $\alpha_{\imath} \in C\left[R_{+}, R_{+}\right], \quad \beta_{\imath} \in C\left[\mathscr{C}_{+}, R_{+}\right], \quad w_{\imath}^{1} \in K$, and $w_{\imath}^{2} \in C\left[\mathscr{C}_{+}, R_{+}\right]$, $w_{l}^{2}(0)=0$, and $w_{\imath}^{2}\left(\sigma^{l}\right)$ is nondecreasing in $\sigma^{l}$ for $\sigma^{l} \in \mathscr{C}_{+}^{\prime}$.

$\mathrm{C}_{2}$. Assume that the interconnection functions $H_{t}$ in (2) satisfy the following constraints: for $(t, \phi) \in R_{+} \times C_{\rho}$

$$
\left\|H_{i}(t, \phi(0), \phi)\right\| \leqq \sum_{j=1}^{m}\left[\bar{e}^{-\jmath} \alpha_{\imath j}(V(t, x)) w_{j}^{\prime}\left(V_{\jmath}\left(t, \phi^{\prime}(0)\right)\right)\right.
$$

$$
\left.+\left\|\bar{\ell}_{t}^{i j}\right\|_{0} \beta_{i j}\left(V_{t}\right) w_{J}^{2}\left(V_{t}^{\prime}\right)\right]
$$

where $\bar{e}^{i j}, \bar{\ell}_{t}^{\prime \prime}$ are elements of the $m \times m$ fundamental interconnection matrices $\bar{E}, \bar{L}_{t} ; \alpha_{i j} \in C\left[R_{+}, R_{+}\right], \alpha_{i j}(u)$ for all $i, j$ are nondecreasing in $u_{\ell}$ 
for $\ell \neq i$, and $\beta_{i j} \in C\left[\mathscr{C}_{+}^{m}, R_{+}\right], \beta_{i j}(\sigma)$ are nondecreasing in $\sigma \in \mathscr{C}_{+}^{m} ; \boldsymbol{w}_{\jmath}^{1}$, $w_{\text {, }}^{2}$ are defined in $\mathrm{C}_{1}(\mathrm{iv})$; and

$$
V(t, \phi(0))=\left(V_{1}\left(t, \phi^{1}(0)\right), V_{2}\left(t, \phi^{2}(0)\right), \cdots, V_{m}\left(t, \phi^{m}(0)\right)\right)^{T} .
$$

By $\bar{A}(\sigma(0))$ and $\bar{B}(\sigma)$, we denote the $m \times m$ matrix function and functional on $R_{+}^{m}$ and $\mathscr{C}_{+}^{m}$ into $R_{+}^{m 2}$ with the coefficients

$$
\bar{a}_{\imath j}(\sigma(0))= \begin{cases}-\alpha_{i}\left(\sigma^{\prime}(0)\right)+K_{i} \bar{e}^{i i} \alpha_{i i}(\sigma(0)), & i=j \\ K_{i} e^{i j} \alpha_{\imath j}(\sigma(0)), & i \neq j\end{cases}
$$

and

$$
\bar{b}_{i j}(\sigma)= \begin{cases}\beta_{l}\left(\sigma^{i}\right)+K_{t}\left\|\bar{\ell}_{t}^{i}\right\|_{0} \beta_{i i}(\sigma), & i=j \\ K_{t}\left\|\bar{\ell}_{t}^{i j}\right\|_{0} \beta_{l j}(\sigma), & i \neq j,\end{cases}
$$

respectively, where $\alpha_{\imath}, \beta_{\imath}, \alpha_{i l}, \beta_{i,}, \bar{e}^{i j}, \bar{\ell}^{i j}$ as defined before.

We note that the conditions $\left(\mathrm{C}_{1}\right)$ and $\left(\mathrm{C}_{2}\right)$ as outlined above can be considered as extension of the decomposition-aggregation technique [ 2 , $8,9]$ for functional differential systems.

Consider the comparison system

$$
\dot{u}=\bar{A}(u) w^{1}(u)+\bar{B}\left(u_{t}\right) w^{2}\left(u_{t}\right)=g\left(t, u, u_{t}\right),
$$

where $u \in R_{+}^{m}, \bar{A}(u)$ and $\bar{B}\left(u_{t}\right)$ are defined in (26) and (27); $w^{1}(u)$ and $w^{2}(u)$ are defined by

$$
\begin{aligned}
w^{1}(u) & =\left(w_{1}^{1}\left(u^{1}\right), w_{2}^{1}\left(u^{2}\right), \cdots w_{m}^{1}\left(u^{m}\right)\right)^{T}, w^{2}\left(u_{t}\right) \\
& =\left(w_{1}^{2}\left(u_{t}^{1}\right), \cdots w_{m}^{2}\left(u_{t}^{m}\right)\right)^{T} .
\end{aligned}
$$

Under the conditions $\left(C_{1}\right)$ and $\left(C_{2}\right)$, we prove the following:

THEOREM 4. The trivial solution $x \equiv 0$ of (1) is connectively stable or connectively asymptotically stable, if the comparison system (28) is stable or asymptotically stable.

Proof. Let us consider the vector Lyapunov function $V(t, \phi(0))$ as defined in (25). It is obvious that it is continuous on $\left[R_{+} \times S_{\rho}, R_{+}^{m}\right]$ and Lipschitzian in $\phi(0)$ for fixed $t \in R_{+}$. From (22), we have

$$
a(\|x\|) \leqq \sum_{i=1}^{m} V_{i}(t, x) \leqq b(\|x\|), \quad(t, x) \in R_{+} \times S_{\rho}
$$


where

$$
a(\|x\|)=\sum_{i=1}^{m} a_{t}\left(\left\|x^{\imath}\right\|\right), \quad b(\|x\|)=\sum_{i=1}^{m} b_{l}\left(\left\|x^{\imath}\right\|\right)
$$

Now, by using (21), (23), (24), (25), (26), (27) and (29) for $t \in R_{+}, \phi \in C_{\rho}$, we compute

$$
\begin{aligned}
& \left.D_{(1)}^{+} V_{\imath}\left(t, \phi^{\prime}(0), \phi\right)\right)=\lim _{h \rightarrow 0^{+}} \sup \frac{1}{h}\left[V_{i}\left(t+h, \phi^{\prime}(0)+h f_{\imath}(t, \phi(0), \phi)\right)\right. \\
& \left.-V_{l}\left(t, \phi^{\prime}(0)\right)\right] \\
& \leqq \lim _{h \rightarrow 0^{+}} \sup \frac{1}{h}\left[V_{\imath}\left(t+h, \phi^{\prime}(0)+h h_{\imath}\left(t, \phi^{\prime}(0), \phi^{\prime}\right)\right)\right. \\
& \left.-V\left(t, \phi^{\prime}(0)\right)\right] \\
& +\lim _{h \rightarrow 0^{+}} \sup \frac{1}{h}\left[V_{\imath}\left(t+h, \phi^{\prime}(0)+h f_{\iota}(t, \phi(0), \phi)\right)\right. \\
& \left.-V_{\imath}\left(t+h, h h_{\imath}\left(t, \phi^{\prime}(0), \phi^{\prime}\right)\right)\right] \\
& \leqq D_{(5)}^{+} V_{\iota}\left(t, \phi^{\prime}(0), \phi^{\imath}\right)+K_{\imath}\left\|H_{\imath}(t,(0), \phi)\right\| \\
& \leqq \sum_{j=1}^{n}\left[\bar{a}_{l j}\left(V(t, \phi(0)) w_{j}^{\prime}\left(V_{l}\left(t, \phi^{\jmath}(0)\right)\right)+\bar{b}_{i j}\left(V_{t}\right) w_{j}^{2}\left(V_{t}^{\jmath}\right)\right]\right. \text {, }
\end{aligned}
$$

for $i \in I,(t, \phi) R_{+} \times C_{\rho}$ which is equivalent to

$$
D_{(1)}^{+} V(t, \phi(0), \phi) \leqq g\left(t, V(t, \phi(0)), V_{\imath}\right)
$$

where

$$
g\left(t, V\left(t, \phi(0), V_{t}\right)=\bar{A}(V(t, \phi(0))) w^{1}\left(V(t, \phi(0))+\bar{B}\left(V_{t}\right) w^{2}\left(V_{t}\right)\right.\right.
$$

By applying the comparison Theorem 1, we obtain

$$
V\left(t, x\left(t_{0} \phi_{0}\right)(t)\right) \leqq r\left(t_{0} \sigma_{0}\right)(t), \quad t \geqq t_{0}
$$

whenever

$$
V_{t 0} \leqq \sigma_{0}
$$

Now from the hypothesis that the trivial solution of (28) is stable or asymptotically stable, we can conclude the connective stability or connec- 
tive asymptotic stability by the application of Theorem 3. This completes the proof of the Theorem.

One may think that Theorem 4 looks like perturbation theorems given by Lakshmikantham and Leela in [6] and by Halanay [3]. However, the difference is obvious. In fact, it is easy to see that for each fixed $i \in I$, the subsystem (2) can not be treated as perturbation (in the sense described in $[3, \mathbf{6}]$ ) of the corresponding isolated subsystem (4). Moreover, earlier perturbation results $[3,6]$ are inadequate for studying such cases. In addition to this, we have made use of vector Lyapunov functions that are more advantageous over single Lyapunov functions. For further usefulness of vector Lyapunov functions over single Lyapunov functions, see Lakshmikantham [5], Lakshmikantham and Leela [6], and Ladde [4, 11].

In this work, we have not shown the usefulness of the concept of connective stability, the role of interconnection matrices, the stability and other properties of the hereditary systems, and its further applications. However, these results are under study and will be reported elsewhere.

\section{REFERENCES}

1. F. N. Bailey, The application of Lyapunov's second method to interconnected systems, J. SIAM Control, Ser. A., 3 (1966), 433-462.

2. L. J. T. Grujic and D. D. Šiljak, Asymptotic stability and instability of large-scale systems, IEEE Trans. AC-18 (1973), 636-645.

3. A. Halanay, Differential Equations, Stability, Oscillation, Time-Lag, Academic Press, New York (1966).

4. G. S. Ladde, Systems of differential inequalities and stochastic differential equations II, J. Math. Phy., 16 (1975), 894-900.

5. V. Lakshmikantham, Vector Lyapunov functions and conditional stability, J. Math. Anal. Appl., 10 (1965), 368-377.

6. V. Lakshmikantham and S. Leela, Differential and Integral Inequalities, Vol. I and II, Academic Press, New York (1969).

7. V. Lakshmikantham and G. R. Shendge, Functional differential inequalities, An. Acad. Brasil, Ci., 39 (1967), 7-14.

8. D. D. Šiljak, Stability of large-scale systems under structural perturbations, IEEE Trans., SMC-2 (1972), 657-663.

9. - On stability of large-scale systems under structural perturbances, IEEE Trans., SMC-3 (1973), 415-417.

10. G. R. Shendge, Finite systems of functional differential inequalities and minimax solutions, Acta Math., Acad. Sci. Hungary, 25 (1974), 21-30.

11. G. S. Ladde, Stability of large -scale hereditary systems under structural perturbations, Proc. of the IFAC Symp. on Large Scale Systems Theory and Applications, Udine, Italy (1976), 215-226.

Received November 4, 1975. The research reported herein was supported by SUNY Research Foundation Faculty Fellowship. 




\section{Pacific Journal of Mathematics}

\section{Vol. 66, No. $1 \quad$ November, 1976}

Helen Elizabeth. Adams, Factorization-prime ideals in integral domains ............ Patrick Robert Ahern and Robert Bruce Schneider, The boundary behavior of Henkin's kernel.

Daniel D. Anderson, Jacob R. Matijevic and Warren Douglas Nichols, The Krull

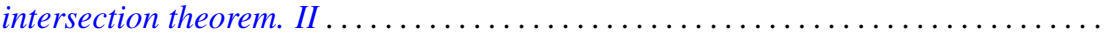

Efraim Pacillas Armendariz, On semiprime P.I.-algebras over commutative regular

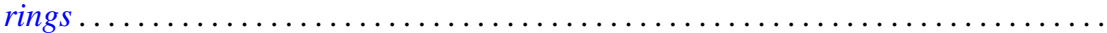

Robert H. Bird and Charles John Parry, Integral bases for bicyclic biquadratic fields

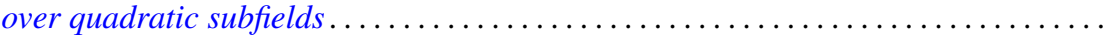

Tae Ho Choe and Young Hee Hong, Extensions of completely regular ordered

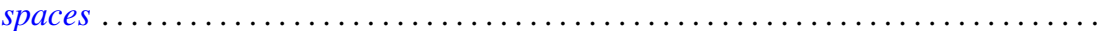

John Dauns, Generalized monoform and quasi injective modules ...............

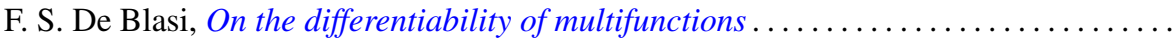

Paul M. Eakin, Jr. and Avinash Madhav Sathaye, R-endomorphisms of $R[[X]]$ are

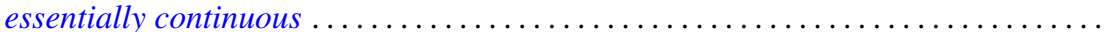

Larry Quin Eifler, Open mapping theorems for probability measures on metric

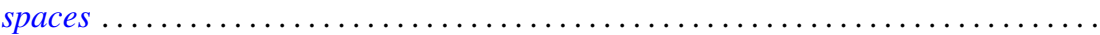

Garret J. Etgen and James Pawlowski, Oscillation criteria for second order self adjoint

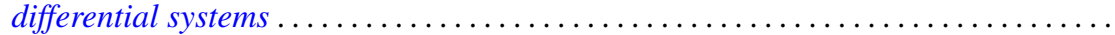

Ronald Fintushel, Local $S^{1}$ actions on 3-manifolds .

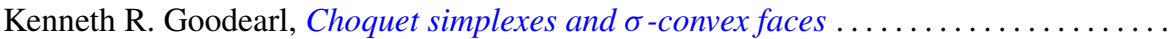

John R. Graef, Some nonoscillation criteria for higher order nonlinear differential

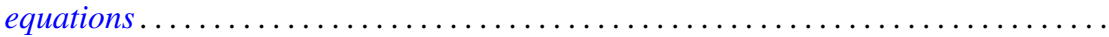

Charles Henry Heiberg, Norms of powers of absolutely convergent Fourier series: an example.

Les Andrew Karlovitz, Existence of fixed points of nonexpansive mappings in a space

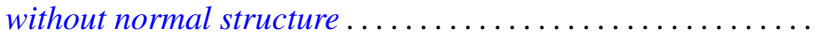

Gangaram S. Ladde, Systems of functional differential inequalities and functional

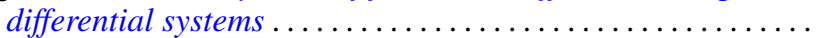

Joseph Michael Lambert, Conditions for simultaneous approximation and interpolation with norm preservation in $C[a, b]$.

Ernest Paul Lane, Insertion of a continuous function.

Robert F. Lax, Weierstrass points of products of Riemann surfaces .

Dan McCord, An estimate of the Nielsen number and an example concerning the Lefschetz fixed point theorem...

Paul Milnes and John Sydney Pym, Counterexample in the theory of continuous functions on topological groups...

Peter Johanna I. M. De Paepe, Homomorphism spaces of algebras of holomorphic functions

Judith Ann Palagallo, A representation of additive functionals on $L^{p}$-spaces,

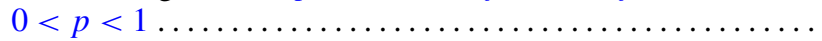

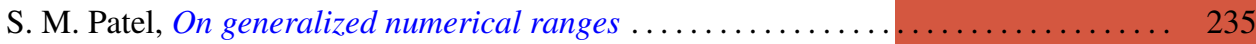

Thomas Thornton Read, A limit-point criterion for expressions with oscillatory

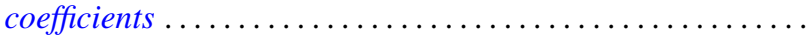

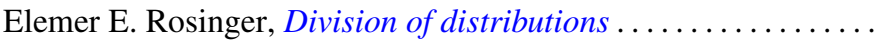

Peter S. Shoenfeld, Highly proximal and generalized almost finite

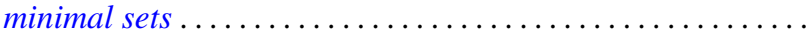

R. Sirois-Dumais and Stephen Willard, Quotient-universal sequential spaces

Robert Charles Thompson, Convex and concave functions of singular values of matrix sums....

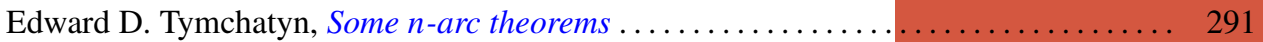

